\title{
A stream multimetric fish index in a large-sized city in south- eastern Brazil
}

\author{
Índice multimétrico de peixes de riachos em uma cidade de grande porte no Sudeste do \\ Brasil
}

\section{Guilherme Raphael Camargo Arcanjo Silva ${ }^{1}$ (D) and Maurício Cetra ${ }^{2 *}$ (D)}

\author{
${ }^{1}$ Programa de Pós-graduação em Sustentabilidade na Gestão Ambiental - PPGSGA, Universidade \\ Federal de São Carlos - UFSCar, Rodovia João Leme dos Santos, Km 110 - SP-264, Sorocaba, SP, \\ Brasil \\ ${ }^{2}$ Departamento de Ciências Ambientais - DCA, Universidade Federal de São Carlos - UFSCar, \\ Rodovia João Leme dos Santos, Km 110 - SP-264, Sorocaba, SP, Brasil \\ *e-mail: mcetra@ufscar.br
}

Cite as: Silva, G.R.C.A. and Cetra, M. A stream multimetric fish index in a large-sized city in southeastern Brazil. Acta Limnologica Brasiliensia, 2021, vol. 33, e14.

\begin{abstract}
Aim: Our study was carried out to develop a multimetric index suitable for urban wadeable streams in Sorocaba, a large-sized city from the Atlantic rainforest in south-eastern Brazil. Methods: Twenty-seven stream stretches were selected for environmental and fish evaluation. Twenty ecological metrics were tested over an environmental gradient between the reference and degraded stretches. Candidate metrics were screened for range, responsiveness, and redundancy. We calculated a multimetric fish index (MFI) subdivided into five quality classes: reference $\geq 0.8,0.6 \leq \operatorname{good}<0.8$, $0.4 \leq$ moderate $<0.6,0.2 \leq$ poor $<0.4$, and bad $<0.2$. Results: Four metrics were adequate for discriminating higher biotic quality from degraded stretches. Five stream stretches (18\%) were classified as a reference or good, and $16(60 \%)$ were poor or bad. Three reference stretches could be used for a hydromorphological restoration programme. Conclusion: Our results indicated that biological integrity was altered, which was indicative of severe environmental degradation. Our study results may be useful for a management and restoration project of the Sorocaba/Médio Tietê hydrographic basin.
\end{abstract}

Keywords: urban streams; habitat quality; Index of Biotic Integrity.

Resumo: Objetivo: Nosso estudo foi realizado para desenvolver um índice multimétrico adequado para riachos urbanos em Sorocaba, uma cidade de grande porte inserida na Mata Atlântica do sudeste do Brasil. Métodos: Vinte e sete trechos de riachos foram selecionados para avaliaçáo ambiental e de peixes. Vinte métricas ecológicas foram testadas em um gradiente ambiental entre os trechos de referência e degradados. As métricas candidatas foram selecionadas quanto à amplitude, capacidade de resposta e redundância. Calculamos um índice multimétrico de peixes (MFI) subdividido em cinco classes de qualidade: referência $\geq 0,8,0,6 \leq$ bom $<0,8,0,4 \leq$ moderado $<0,6,0,2 \leq$ pobre $<0,4$ e ruim < 0,2. Resultados: Quatro métricas se mostraram adequadas para discriminar trechos com maior qualidade biótica daqueles degradados. Cinco trechos de riacho (18\%) foram classificados como referência ou bom e 16 (60\%) como pobre ou ruim. Três trechos de referência podem ser usados para um programa de restauração hidromorfológica. Conclusóes: Esses resultados indicaram que muitos aspectos da integridade biológica foram alterados, indicativo de severa degradação. Nossos resultados podem ser úteis para um projeto de gestão e restauração da bacia hidrográfica do Sorocaba/Médio Tietê.

Palavras-chave: riachos urbanos; qualidade do habitat; Índice de Integridade Biótica. 


\section{Introduction}

Stressors can be considered as any physical, chemical or biological parameters or entities that directly or indirectly result in biotic responses of concern (USEPA, 2017). Urbanization can cause perturbations to a system through sewage effluent, channelization, and riparian vegetation reduction. These multiple stressors can make it difficult to determine individual stressor relative importance (Paul \& Meyer, 2001; Walsh et al., 2005).

Urbanization-induced changes in water quality and or hydrologic regimes are referred to as urban stream syndrome (Sheldon et al., 2019). The loss of breeding, feeding, and resting habitat can modify the fish community. These mechanistic pathways are not well studied in urban streams (Wenger et al., 2009). The fish assemblage similarity of urban with reference streams, species richness and diversity, proportions of lithophilic spawners fish, and sensitive species decreased with increasing urbanization (Helms et al., 2005; Wang et al., 2001; Morgan \& Cushman, 2005). On the other hand, tolerant species, fish with eroded fins, lesions, tumours increased with urbanization (Helms et al., 2005; Morgan \& Cushman, 2005). The amount of connected impervious surface may have harsh effects on fish assemblages in the river basin scale, including low fish density, species richness, diversity, and index of biotic integrity (IBI) score (Wang et al. 2001). On the other hand, historical species pool and stream characteristics can shape fish communities, with urbanization playing a rather individual role (Czeglédi et al., 2020).

Biological monitoring can be an essential tool for properly managing water resources, reflecting physical, chemical and biological stream structures (Karr, 1991). The multimetric approach (Karr, $1981)$ attempts to provide an integrated analysis of the biological community (e.g., species richness, diversity indices, and feeding type composition) into a unitless measure, which can be used to assess an overall site condition (Hering et al., 2006). There is no universal multimetric index (Davis and Simon, 1995). One must consider the intrinsic regional characteristics to select a set of biological variables used to categorize local environmental changes (Omernik, 1995).

Several studies have adapted a multimetric index based on neotropical fish assemblages (Bozzetti \& Schulz, 2004; Marciano et al., 2004; Ferreira \& Casatti, 2006; Casatti et al., 2009; Esteves \& Alexandre, 2011; Casatti et al., 2012; Terra et al.,
2013; Santos \& Esteves, 2015; Cetra \& Ferreira, 2016; Gonino et al., 2020).

The relationships between the fish community and environmental perturbations can be obtained using a multimetric biotic index and physical habitat structure assessment. The structure of the surrounding physical habitat (e.g., canopy cover, riparian vegetation structure complexity, and a measure of disturbance) that influences the quality of the water resource (e.g., substratum mean diameter and stability, large woody debris and proportion of reach composed of pools and riffles) compound the physical habitat structure assessment (Barbour et al., 1999).

Our objective was to develop a multimetric index suitable for urban wadeable streams in the Atlantic rainforest of south-eastern Brazil. We selected fish richness, abundance, trophic structure, and habitat use metrics. We understand how physical habitat disturbances influence different aspects of the fish communities, i.e., which species and or which metrics are correlated with disturbance gradients. By these analyses, we aim to develop a tool to diagnose the urban streams state. The methods used in our study may be helpful in a management and restoration hydrographic basin programme.

\section{Materials and Methods}

\subsection{Study area}

This study was conducted in the City of Sorocaba $\left(23^{\circ} 20^{\prime}-23^{\circ} 35^{\prime} S ; 47^{\circ} 17^{\prime}-47^{\circ} 34^{\prime} \mathrm{W}\right)$ in the south-east of Brazil, in São Paulo state. The Sorocaba river basin presents intense urban, industrial, and agricultural activity. In the middle of the 17th century, Sorocaba municipality developed along the Sorocaba River. In the second half of the 18 th century, several districts already existed. From the 1950s, the river was rectified, and between 1960 and 1990, the pollution from industrial activities was intense. The worst fish mortality occurred in 1978, in which tons of fish perished over a large stretch of the river. Sewage treatment starts at the beginning of the 21st century and gradually recovered the Sorocaba River limnological characteristics (Smith, 2003).

The city of Sorocaba has a total area of $450.4 \mathrm{~km}^{2}$, including $355 \mathrm{~km}^{2}$ of urbanization. The Sorocaba River passes through the town. Sorocaba is the 32nd largest city in Brazil (5570 counties) and 9th in São Paulo (645 counties) in terms of population size, with an estimated total population of 586,625 (population density $1,304.18 \mathrm{~km}^{-2}$ ) (IBGE, 2010). Sorocaba City has a matrix of forest 
patches within the anthropic landscape. Forest covers $17 \%$ of the city area, and $60 \%$ of the forest patches comprise $<1$ ha (Mello et al., 2016). The average yearly rainfall within the basin is around $1300 \mathrm{~mm}$, with the most massive fall in the summer. The amount of precipitation and its intensity varies considerably on a seasonal basis, with a drier season occurring from April to August $\left(12\right.$ to $22^{\circ} \mathrm{C}$ ) and a wetter period in September to March (16 to $\left.27^{\circ} \mathrm{C}\right)$. The region is characterized by transitional vegetation between the Atlantic Forest and the Cerrado (Mello et al., 2016).

\subsection{Sampling sites}

Twenty-seven wadeable stream stretches were investigated during the dry season in 2016 (Figures 1 and 2). Twenty-three stretches were in Sorocaba City and four on the edge of the Floresta Nacional de Ipanema (FLONA-Ipanema), which is without the direct influence of urbanization and are our reference stream stretches. This design created a disturbance gradient that is important in developing a multimetric index (Hughes et al., 1998). The stream stretches measured $3.4 \pm 1.7 \mathrm{~m}$ in width and $26.7 \pm 21.0 \mathrm{~cm}$ in depth (mean \pm S.D.).

\subsection{Stream stretches classification}

To characterize the stream stretches, we used a physical habitat index (PHI) (Barbour et al., 1999). We evaluated the stretches with nine habitat parameters: epifaunal substratum available cover, velocity/depth regime, sediment deposition, channel flow status, channel alteration, frequency of riffles, bank stability, vegetative protection, and riparian vegetative zone width (Table 1 ). The PHI range classification was: 0 to 45 (poor), 46 to 90 (marginal), 91 to 135 (suboptimal) and 136 to 180 (optimal).

\subsection{Fish collection}

Seasonal fluctuation of the water level is one of the most important factors influencing the structure of fish assemblages (Rodríguez \& Lewis Junior, 1997). The sampling period for the ichthyofauna was the dry season. During this period, connections between the structure of the fish assemblage and the habitat structure are more robust, the effect of temporal variation can be controlled, and sampling is more efficient due to the smaller volume of water and the consequent increase in fish density (Willis et al., 2005; Pease et al., 2012).

We used data from single-pass electric fishing catches (Fame Consortium, 2004) performed using an LR-24 Smith Root backpack between 0800 and 1700 hours without stop nets at the upper and lower stream stretches limits (License no 13352-1 SISBIO/ IBAMA/MMA). The ichthyofauna was collected from $70 \mathrm{~m}$ stretches representing the range of available mesohabitats, i.e., a repeating sequence of a riffle, pool and run.

Vouchers of the species collected were deposited in the collection of Laboratório de Ictiologia de

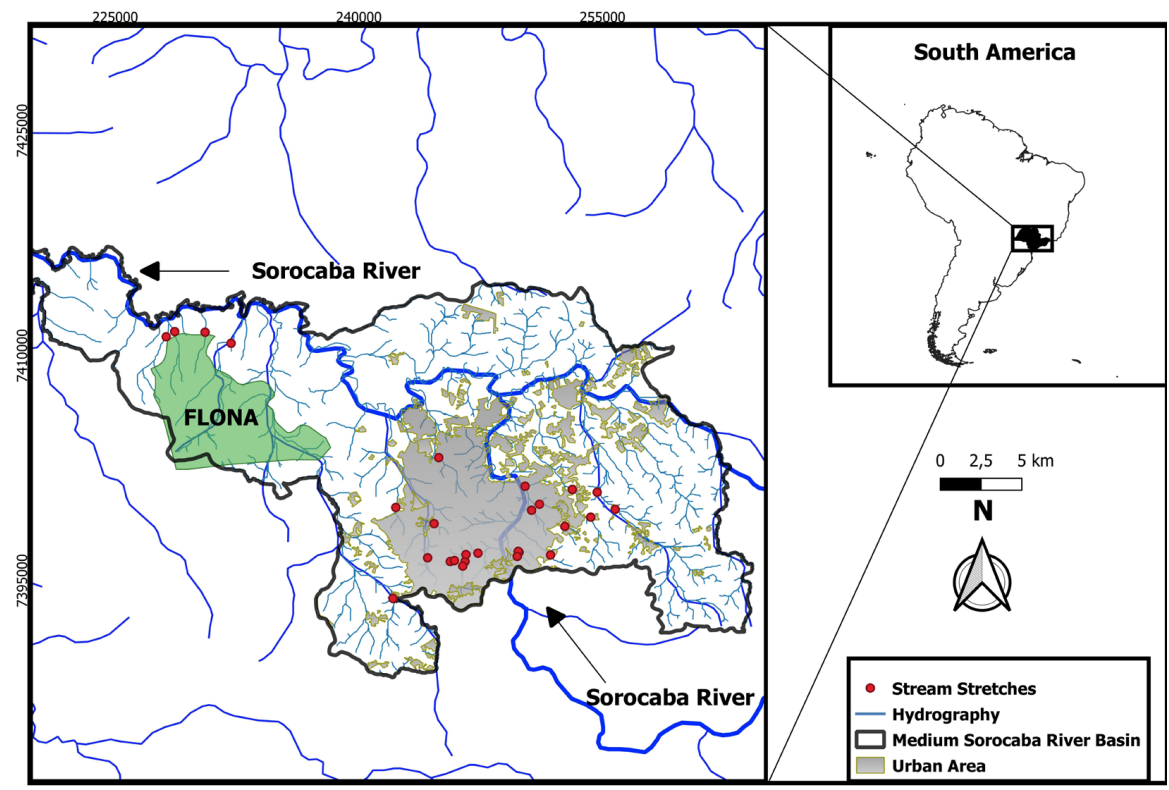

Figure 1. State of São Paulo, Medium Sorocaba River Basin and stream stretches sampled in Sorocaba City and FLONA-Ipanema (Datum SAD 69, UTM 23S). 

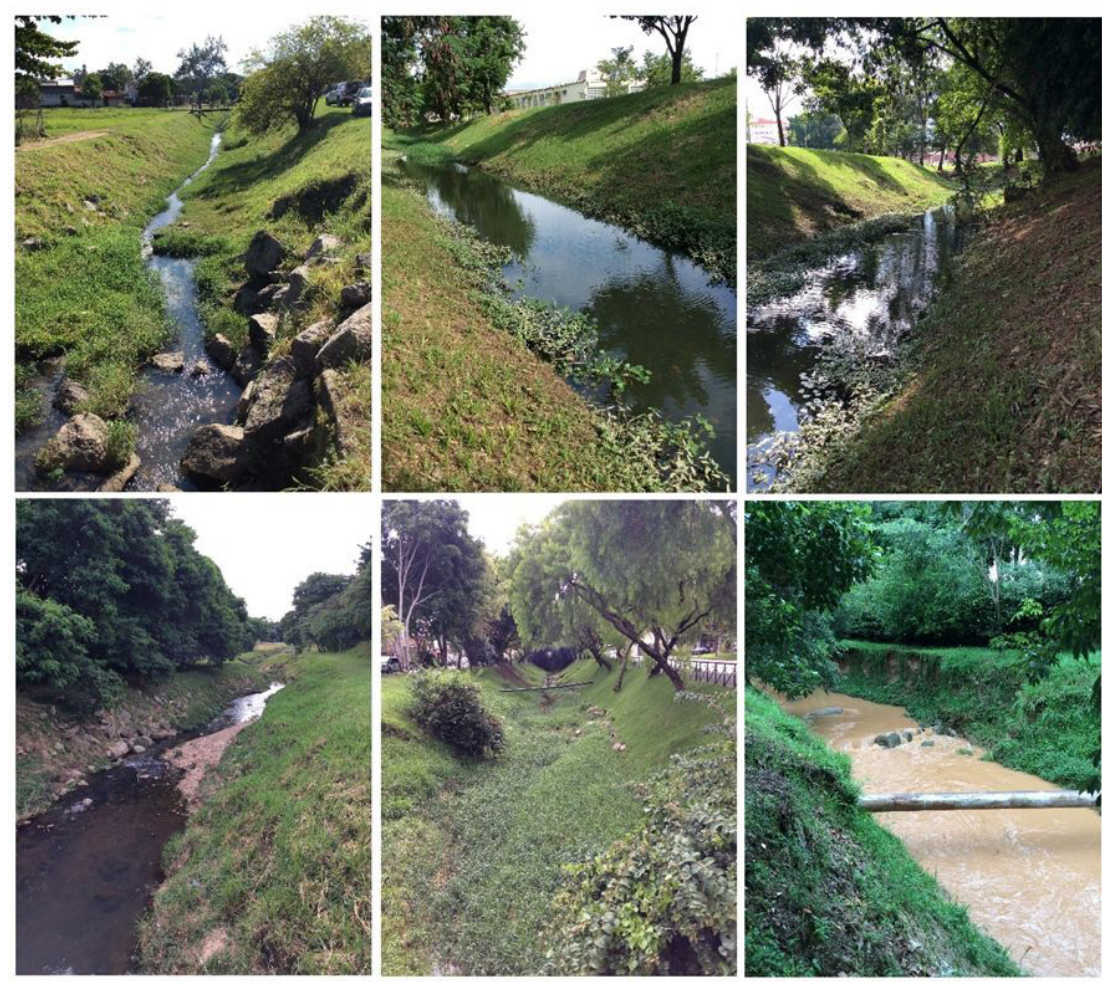

Figure 2. Representative stream stretches sampled from Sorocaba City.

Sorocaba (LISO-UFSCar-Sorocaba). The specimens were identified by Prof. Dr. George Mendes Taliaferro Mattox (UFSCar-Sorocaba).

\subsection{Data analysis}

Species were categorized into a trophic group and position in the water column (Casatti et al., 2012) (Table 2). Twenty metrics were considered (Table 3 ). The metrics were grouped into richness and origin, abundance, trophic structure, and habitat use. The assumption underpinning the species richness and origin category is that environmental degradation will change communities containing many species to simple assemblages dominated by a few species (Barbour et al., 1999). The proportion of exotic species measures the extent to which introduced species have invaded the fish assemblage. The presence of exotic species reflects biological pollution, and generally, these species are more tolerant of degradation of habitat and water quality than the native species and thus may indicate degraded conditions (Barbour et al., 1999). A healthy and stable assemblage will be relatively consistent in its proportional representation. The relative contribution of the populations (e.g., Cyprinodontiformes) to the total fauna is a simple measure of redundancy, and a high level of redundancy is equated with the dominance of a pollution tolerant organism and a lowered diversity.
Trophic structure measures provide information on the balance of feeding strategies. Without relatively stable food dynamics, an imbalance in functional feeding groups will result, reflecting stressed conditions. Insectivores are the dominant trophic guild of most tropical streams (Winemiller et al., 2018). As the invertebrate food source decreases in abundance and diversity due to habitat degradation by urbanization, there is a shift from insectivorous to omnivorous fish species (Barbour et al., 1999). The habitat use category metrics were used to make the index sensitive to stream geomorphology changes resulting from the effects of channelization and dams on habitats required by benthic riffle and water column species.

Candidate metrics were screened for range, responsiveness, and redundancy. First, a principal component analysis (PCA) was used to detect the metrics with low variance. We used a brokenstick model to decide which axes were important and representative. Metrics with a factor loading $>0.6$ were rejected. Secondly, a Pearson correlation coefficient $(r)$ significance $(\alpha<0.10)$ was used to examine the responsiveness of the remaining candidate metrics discriminating the minimally and the most disturbing sites based on the PHI. Thirdly, $r$ was used to test redundancy. Pairs of the metrics with strong positive correlations $(r>0.75)$ were considered redundant. The metrics were then 
Table 1. Habitat parameters, condition categories and scores of the sampled streams from Sorocaba City (adapted from Barbour et al., 1999).

\begin{tabular}{|c|c|c|c|c|}
\hline \multirow{2}{*}{$\begin{array}{c}\text { Habitat } \\
\text { parameter }\end{array}$} & \multicolumn{4}{|c|}{ Condition category } \\
\hline & Optimal & Suboptimal & Marginal & Poor \\
\hline $\begin{array}{c}\text { Epifaunal } \\
\text { substratum/ } \\
\text { available cover }\end{array}$ & $\begin{array}{c}\text { Greater than } 70 \% \text { of } \\
\text { substratum favourable for } \\
\text { epifaunal colonization and } \\
\text { fish cover; mix of snags, } \\
\text { submerged logs, undercut } \\
\text { banks, cobble or other } \\
\text { stable habitat and at a stage } \\
\text { to allow full colonization } \\
\text { potential (i.e., logs and } \\
\text { snags that are not new fall } \\
\text { nor transient). }\end{array}$ & $\begin{array}{l}40-70 \% \text { mix of } \\
\text { stable habitat; } \\
\text { well-suited for } \\
\text { full colonization } \\
\text { potential; } \\
\text { adequate habitat } \\
\text { for maintenance } \\
\text { of populations; } \\
\text { presence of } \\
\text { additional } \\
\text { substratum in the } \\
\text { form of new-fall, but } \\
\text { not yet prepared for } \\
\text { colonization (may } \\
\text { rate at high end of } \\
\text { scale). }\end{array}$ & $\begin{array}{c}20-40 \% \text { mix of } \\
\text { stable habitat; } \\
\text { habitat availability } \\
\text { less than desirable; } \\
\text { substratum } \\
\text { frequently disturbed } \\
\text { or removed. }\end{array}$ & $\begin{array}{l}\text { Less than } 20 \% \text { stable } \\
\text { habitat; lack of habitat } \\
\text { is obvious; substratum } \\
\text { unstable or lacking. }\end{array}$ \\
\hline Score & $\begin{array}{llll}19 & 18 & 17 & 16\end{array}$ & $\begin{array}{lllll}15 & 14 & 13 & 12 & 11\end{array}$ & 109 & 32 \\
\hline $\begin{array}{l}\text { Velocity/depth } \\
\text { regime }\end{array}$ & $\begin{array}{l}\text { All four velocity: depth } \\
\text { regimes present (slow-deep, } \\
\text { slow-shallow, fast-deep, } \\
\text { fast-shallow). (Slow is }<0.3 \\
\left.\mathrm{~ms}^{-1} \text {, deep is }>0.5 \mathrm{~m}\right)\end{array}$ & $\begin{array}{l}\text { Only three of } \\
\text { the four regimes } \\
\text { present (if fast- } \\
\text { shallow is missing, } \\
\text { score lower than } \\
\text { if missing other } \\
\text { regimes). }\end{array}$ & $\begin{array}{l}\text { Only two of the four } \\
\text { habitat regimes } \\
\text { present (if fast- } \\
\text { shallow or slow- } \\
\text { shallow are missing, } \\
\text { score low). }\end{array}$ & $\begin{array}{l}\text { Dominated by one } \\
\text { velocity/depth regime } \\
\text { (usually slow-deep). }\end{array}$ \\
\hline Score & $\begin{array}{llll}19 & 18 & 17 & 16\end{array}$ & $\begin{array}{lllll}15 & 14 & 13 & 12 & 11\end{array}$ & $\begin{array}{lllll}10 & 9 & 8 & 7 & 6\end{array}$ & $\begin{array}{llllll}5 & 4 & 3 & 2 & 1 & 0\end{array}$ \\
\hline $\begin{array}{c}\text { Sediment } \\
\text { deposition }\end{array}$ & $\begin{array}{l}\text { Little or no enlargement of } \\
\text { islands or point bars and < } \\
5 \% \text { of the bottom affected by } \\
\text { sediment deposition }\end{array}$ & $\begin{array}{l}\text { Some new increase } \\
\text { in bar formation, } \\
\text { mostly from gravel, } \\
\text { sand, or fine } \\
\text { sediment; } 5-30 \% \text { of } \\
\text { the bottom affected; } \\
\text { slight deposition in } \\
\text { pools. }\end{array}$ & $\begin{array}{l}\text { Moderate deposition } \\
\text { of new gravel, sand, } \\
\text { or fine sediment } \\
\text { on old and new } \\
\text { bars; } 30-50 \% \text { of the } \\
\text { bottom affected; } \\
\text { sediment deposits } \\
\text { at obstructions, } \\
\text { constrictions and } \\
\text { bends; moderate } \\
\text { deposition of pools } \\
\text { prevalent. }\end{array}$ & $\begin{array}{l}\text { Heavy deposits of fine } \\
\text { material, increased bar } \\
\text { development; > 50\% } \\
\text { of the bottom changing } \\
\text { frequently; pools } \\
\text { almost absent due to } \\
\text { substantial sediment } \\
\text { deposition. }\end{array}$ \\
\hline Score & $\begin{array}{llll}19 & 18 & 17 & 16\end{array}$ & $\begin{array}{lllll}15 & 14 & 13 & 12 & 11\end{array}$ & $\begin{array}{lllll}10 & 9 & 8 & 7 & 6\end{array}$ & $\begin{array}{llllll}5 & 4 & 3 & 2 & 1 & 0\end{array}$ \\
\hline $\begin{array}{c}\text { Channel flow } \\
\text { status }\end{array}$ & $\begin{array}{l}\text { Water reaches base of } \\
\text { both lower banks, and } \\
\text { minimal amount of channel } \\
\text { substratum is exposed. }\end{array}$ & $\begin{array}{l}\text { Water fills }>75 \% \\
\text { of the available } \\
\text { channel; or }<25 \% \text { of } \\
\text { channel substratum } \\
\text { is exposed. }\end{array}$ & $\begin{array}{l}\text { Water fills } 25-75 \% \\
\text { of the available } \\
\text { channel, and/or riffle } \\
\text { substrata are mostly } \\
\text { exposed. }\end{array}$ & $\begin{array}{l}\text { Very little water in } \\
\text { channel and mostly } \\
\text { present as standing } \\
\text { pools. }\end{array}$ \\
\hline Score & $\begin{array}{llll}19 & 18 & 17 & 16\end{array}$ & $\begin{array}{lllll}15 & 14 & 13 & 12 & 11\end{array}$ & $\begin{array}{lllll}10 & 9 & 8 & 7 & 6\end{array}$ & $\begin{array}{llllll}5 & 4 & 3 & 2 & 1 & 0\end{array}$ \\
\hline $\begin{array}{l}\text { Channel } \\
\text { alteration }\end{array}$ & $\begin{array}{l}\text { Channelization or dredging } \\
\text { absent or minimal; stream } \\
\text { with normal pattern. }\end{array}$ & $\begin{array}{c}\text { Some } \\
\text { channelization } \\
\text { present, usually } \\
\text { in areas of bridge } \\
\text { abutments; } \\
\text { evidence of past } \\
\text { channelization, } \\
\text { i.e., dredging, } \\
\text { (greater than past } \\
20 \text { years) may be } \\
\text { present, but recent } \\
\text { channelization is not } \\
\text { present. }\end{array}$ & $\begin{array}{l}\text { Channelization } \\
\text { may be extensive; } \\
\text { embankments or } \\
\text { shoring structures } \\
\text { present on both } \\
\text { banks; and } 40 \text { to } \\
80 \% \text { of stream } \\
\text { reach channelized } \\
\text { and disrupted. }\end{array}$ & $\begin{array}{l}\text { Banks shored with } \\
\text { gabion or cement; over } \\
80 \% \text { of the stream } \\
\text { reach channelized and } \\
\text { disrupted. Instream } \\
\text { habitat greatly altered or } \\
\text { removed entirely. }\end{array}$ \\
\hline
\end{tabular}


Table 1. Continued...

\begin{tabular}{|c|c|c|c|c|c|c|c|c|c|c|c|c|c|c|c|}
\hline \multirow{3}{*}{$\begin{array}{c}\begin{array}{c}\text { Habitat } \\
\text { parameter }\end{array} \\
\text { Score }\end{array}$} & \multicolumn{15}{|c|}{ Condition category } \\
\hline & \multicolumn{3}{|c|}{ Optimal } & \multicolumn{3}{|c|}{ Suboptimal } & \multicolumn{4}{|c|}{ Marginal } & \multicolumn{5}{|c|}{ Poor } \\
\hline & 20 & 19 & $\begin{array}{lll}18 & 17 & 16\end{array}$ & 15 & 14 & $\begin{array}{lll}13 & 12 & 11\end{array}$ & 10 & 9 & 8 & 6 & 5 & 4 & 3 & 2 & 10 \\
\hline $\begin{array}{l}\text { Frequency } \\
\text { of riffles (or } \\
\text { bends) }\end{array}$ & \multicolumn{3}{|c|}{$\begin{array}{l}\text { Occurrence of riffles } \\
\text { relatively frequent; ratio of } \\
\text { distance between riffles } \\
\text { divided by width of the } \\
\text { stream }<7: 1 \text { (generally } 5 \\
\text { to } 7 \text { ); variety of habitat is } \\
\text { key. In streams where riffles } \\
\text { are continuous, placement } \\
\text { of boulders or other large, } \\
\text { natural obstruction is } \\
\text { important. }\end{array}$} & \multicolumn{3}{|c|}{$\begin{array}{l}\text { Occurrence of riffles } \\
\text { infrequent; distance } \\
\text { between riffles } \\
\text { divided by the width } \\
\text { of the stream is } \\
\text { between } 7 \text { to } 15 .\end{array}$} & \multicolumn{4}{|c|}{$\begin{array}{l}\text { Occasional riffle } \\
\text { or bend; bottom } \\
\text { contours provide } \\
\text { some habitat; } \\
\text { distance between } \\
\text { riffles divided by the } \\
\text { width of the stream } \\
\text { is between } 15 \text { to } 25 \text {. }\end{array}$} & \multicolumn{5}{|c|}{$\begin{array}{l}\text { Generally, all flat water } \\
\text { or shallow riffles; } \\
\text { poor habitat; distance } \\
\text { between riffles divided } \\
\text { by the width of the } \\
\text { stream is a ratio of }>25 \text {. }\end{array}$} \\
\hline Score & 20 & 19 & $\begin{array}{lll}18 & 17 & 16\end{array}$ & 15 & 14 & $\begin{array}{lll}13 & 12 & 11\end{array}$ & 10 & 9 & 8 & 76 & 5 & 4 & 3 & 2 & 10 \\
\hline $\begin{array}{c}\text { Bank stability } \\
\text { (score each } \\
\text { bank) }\end{array}$ & \multicolumn{3}{|c|}{$\begin{array}{l}\text { Bank stable; evidence of } \\
\text { erosion or bank failure } \\
\text { absent or minimal; little } \\
\text { potential for future problems. } \\
<5 \% \text { of bank affected. }\end{array}$} & \multicolumn{3}{|c|}{$\begin{array}{l}\text { Moderately stable; } \\
\text { infrequent, small } \\
\text { areas of erosion } \\
\text { mostly healed over. } \\
5-30 \% \text { of bank in } \\
\text { reach has areas of } \\
\text { erosion. }\end{array}$} & \multicolumn{4}{|c|}{$\begin{array}{c}\text { Moderately } \\
\text { unstable; } 30-60 \% \text { of } \\
\text { bank in reach has } \\
\text { areas of erosion; } \\
\text { high erosion } \\
\text { potential during } \\
\text { floods. }\end{array}$} & \multicolumn{5}{|c|}{$\begin{array}{l}\text { Unstable; many eroded } \\
\text { areas; 'raw' areas } \\
\text { frequent along straight } \\
\text { sections and bends; } \\
\text { obvious bank sloughing; } \\
60-100 \% \text { of bank has } \\
\text { erosional scars. }\end{array}$} \\
\hline \multirow{2}{*}{ Score } & Left bank & 10 & 9 & 8 & 7 & 6 & 5 & 4 & 3 & & 2 & 1 & 0 & & \\
\hline & Right bank & 10 & 9 & 8 & 7 & 6 & 5 & 4 & 3 & & 2 & 1 & 0 & & \\
\hline $\begin{array}{l}\text { Vegetative } \\
\text { protection } \\
\text { (score each } \\
\text { bank) }\end{array}$ & \multicolumn{3}{|c|}{$\begin{array}{l}\text { More than } 90 \% \text { of the } \\
\text { streambank surfaces } \\
\text { and immediate riparian } \\
\text { zone covered by native } \\
\text { vegetation, including trees, } \\
\text { understory shrubs or } \\
\text { non-woody macrophytes; } \\
\text { vegetative disruption } \\
\text { through grazing or mowing } \\
\text { minimal or not evident; } \\
\text { almost all plants allowed to } \\
\text { grow naturally. }\end{array}$} & \multicolumn{3}{|c|}{$\begin{array}{l}70-90 \% \text { of the } \\
\text { streambank } \\
\text { surfaces covered by } \\
\text { native vegetation, } \\
\text { but one class } \\
\text { of plants is not } \\
\text { well represented; } \\
\text { disruption evident } \\
\text { but not affecting } \\
\text { full plant growth } \\
\text { potential to any } \\
\text { great extent; more } \\
\text { than one-half of } \\
\text { the potential plant } \\
\text { stubble height } \\
\text { remaining. }\end{array}$} & \multicolumn{4}{|c|}{$\begin{array}{l}50-70 \% \text { of the } \\
\text { streambank } \\
\text { surfaces covered } \\
\text { by vegetation; } \\
\text { disruption obvious; } \\
\text { patches of bare soil } \\
\text { or closely cropped } \\
\text { vegetation common; } \\
\text { less than one-half } \\
\text { of the potential } \\
\text { plant stubble height } \\
\text { remaining. }\end{array}$} & \multicolumn{5}{|c|}{$\begin{array}{l}\text { Less than } 50 \% \text { of the } \\
\text { streambank surfaces } \\
\text { covered by vegetation; } \\
\text { disruption of streambank } \\
\text { vegetation is very high; } \\
\text { vegetation has been } \\
\text { removed to } 5 \mathrm{~cm} \text { or } \\
\text { less in average stubble } \\
\text { height }\end{array}$} \\
\hline \multirow{2}{*}{ Score } & Left bank & 10 & 9 & 8 & 7 & 6 & 5 & 4 & 3 & & 2 & 1 & 0 & & \\
\hline & Right bank & 10 & 9 & 8 & 7 & 6 & 5 & 4 & 3 & & 2 & 1 & 0 & & \\
\hline $\begin{array}{l}\text { Riparian } \\
\text { vegetative zone } \\
\text { width (score } \\
\text { each bank } \\
\text { riparian zone) }\end{array}$ & \multicolumn{3}{|c|}{$\begin{array}{l}\text { Width of riparian zone >18 } \\
\text { m; human activities (i.e., } \\
\text { parking lots, roadbeds, } \\
\text { clear-cuts, lawns, or crops) } \\
\text { have not impacted zone. }\end{array}$} & \multicolumn{3}{|c|}{$\begin{array}{l}\text { Width of riparian } \\
\text { zone } 12-18 \mathrm{~m} ; \\
\text { human activities } \\
\text { have impacted zone } \\
\text { only minimally. }\end{array}$} & \multicolumn{4}{|c|}{$\begin{array}{l}\text { Width of riparian } \\
\text { zone } 6-12 \mathrm{~m} ; \\
\text { human activities } \\
\text { have impacted zone } \\
\text { a great deal. }\end{array}$} & \multicolumn{5}{|c|}{$\begin{array}{c}\text { Width of riparian zone } \\
<6 \mathrm{~m} \text { : little or no riparian } \\
\text { vegetation due to human } \\
\text { activities. }\end{array}$} \\
\hline \multirow{2}{*}{ Score } & Left bank & 10 & 9 & 8 & 7 & 6 & 5 & 4 & 3 & & 2 & 1 & 0 & & \\
\hline & Right bank & 10 & 9 & 8 & 7 & 6 & 5 & 4 & 3 & & 2 & 1 & 0 & & \\
\hline
\end{tabular}

selected based on their responsiveness and the applicability to the study area (Casatti et al., 2009; Jia et al., 2013). Proportional variables were arcsinesquare root-transformed, whereas other variables were natural log-transformed before analysis.
The core metrics selected varied between different ranges of values. We normalized the core metrics via transformation to unitless scores to combine these individual measures into an integrated multimetric index. Each metric result was translated into a value 
Table 2. Classification of sampled species according to attributes related to trophic group (alg, algivores; aquins, insectivores with predominance of aquatic forms; car, carnivores; det, detritivores; oni, omnivores; terins, insectivores with predominance of terrestrial forms), position in the water column (ben, benthic; ben/rif, benthic associated with riffles; nek, nekton; nek/bank, nekton associated with stream banks; sur, close to the surface of the water) and origin in the Upper Paraná River system (nat, natives; exo, exotics).

\begin{tabular}{|c|c|c|c|}
\hline Order/species & Trophic & Position & Origin $^{a}$ \\
\hline \multicolumn{4}{|l|}{ Characiformes } \\
\hline Astyanax lacustris (Lütken, 1875) & oni & nek & nat \\
\hline Psalidodon bockmanni (Vari \& Castro 2007) & oni & nek & nat \\
\hline Psalidodon fasciatus (Cuvier, 1819) & terins & nek & nat \\
\hline Deuterodon intermedius (Eigenmann 1908) & terins $^{b}$ & nek & nat \\
\hline Bryconamericus sp. & terins ${ }^{c}$ & nek & nat \\
\hline Hyphessobrycon bifasciatus Ellis, 1911 & terins & nek & nat \\
\hline Serrapinnus notomelas (Eigenmann, 1915) & alg & sur & nat \\
\hline Prochilodus lineatus (Valenciennes, 1837) & $\operatorname{det}^{d}$ & ben & nat \\
\hline Cyphocharax modestus (Fernández-Yépez, 1948) & det & ben & nat \\
\hline Characidium zebra Eigenmann, 1909 & aquins & ben/rif & nat \\
\hline Hoplias malabaricus (Bloch, 1794) & car & nek/ban & nat \\
\hline \multicolumn{4}{|l|}{ Siluriformes } \\
\hline Callichthys callichthys (Linnaeus, 1758) & oni & ben & nat \\
\hline Corydoras aeneus (Gill, 1858) & aquins & ben & nat \\
\hline Hoplosternum littorale (Hancock, 1828) & oni & ben & nat \\
\hline Imparfinis mirini Haseman, 1911 & aquins & ben/rif & nat \\
\hline Pimelodella avanhandavae Eigenmann, 1917 & aquins & ben & nat \\
\hline Rhamdia quelen (Quoy \& Gaimard, 1824) & aquins & ben & nat \\
\hline Hypostomus ancistroides (Ihering, 1911) & det & ben & nat \\
\hline \multicolumn{4}{|l|}{ Gymnotiformes } \\
\hline Gymnotus sylvius Albert \& Fernandes-Matioli, 1999 & aquins & nek/ban & nat \\
\hline \multicolumn{4}{|l|}{ Cyprinodontiformes } \\
\hline Phalloceros harpagos Lucinda, 2008 & oni & sur & nat \\
\hline Poecilia reticulata Peters, 1859 & det & sur & exo \\
\hline \multicolumn{4}{|l|}{ Synbranchiformes } \\
\hline Synbranchus marmoratus Bloch, 1795 & car & nek/ban & nat \\
\hline \multicolumn{4}{|l|}{ Perciformes } \\
\hline Australoheros facetus (Jenyns, 1842) & aquins $^{e}$ & nek/ban & nat \\
\hline Crenicichla britskii Kullander, 1982 & aquins & nek/ban & nat \\
\hline Geophagus brasiliensis (Quoy \& Gaimard, 1824) & oni & ben & nat \\
\hline Oreochromis niloticus (Linnaeus, 1758) & oni & ben & exo \\
\hline
\end{tabular}

${ }^{a}$ Langeani et al. (2007); ${ }^{\mathrm{b}}$ Abilhoa (2007), ${ }^{\mathrm{c}}$ Brandão-Gonçalves et al. (2010); ${ }^{\mathrm{d}}$ Moraes et al. (1997); ${ }^{\mathrm{e}}$ Fernández et al. (2012).

between 0 and 1 (ecological quality ratio, EQR). The EQR represents the relationship between the values of the biological variables observed for a given stream stretch and the values for these variables under the reference conditions applicable to that stream stretch. The ratio is expressed as a numerical value between zero and one: high ecological status is represented by values close to one and low ecological status by values close to zero (Hering et al., 2006).

We used the 'general approach' to calculate the multimetric index (Hering et al., 2006). In the 'general approach', the metrics results are individually compared to the respective metric values under reference conditions. From this comparison, we scored each metric. The multimetric index was a combination of these scores. The same number of metrics has been selected for each metric category and the final multimetric fish index (MFI) was the mean of the $0-1$ digit scores of all core metrics (Böhmer et al., 2004). This range was subdivided into five quality classes using the setting class boundaries (Hering et al., 2006): reference $\geq 0.8$, good $\geq 0.6<0.8$, moderate $\geq 0.4<0.6$, poor $\geq 0.2<0.4$, and bad $<0.2$.

\section{Results}

All habitat parameters varied from poor to optimal. Fifty to sixty per cent of the stream stretches had an epifaunal substratum/available cover, velocity/depth regime, riparian vegetative 
Table 3. Candidate metrics for the index of biotic integrity and the expected response to an increase in environmental degradation.

\begin{tabular}{|c|c|}
\hline Category/candidate metric & Response to environmental degradation \\
\hline \multicolumn{2}{|l|}{ Richness and origin } \\
\hline Species richness (S) & reduces \\
\hline Simpson effective number of species (1/D) & reduces \\
\hline Shannon effective number of species $\left(\mathrm{e}^{\wedge} \mathrm{H}\right)$ & reduces \\
\hline Proportion of exotic species (Psexot) & increases \\
\hline \multicolumn{2}{|l|}{ Abundance } \\
\hline Total abundance $(N)$ & reduces \\
\hline Proportion of exotic species abundance (Pnexot) & increases \\
\hline Proportion of Cyprinodontiformes abundance (PCyp) & increases \\
\hline \multicolumn{2}{|l|}{ Trophic structure } \\
\hline Trophic categories (Stroph) & reduces \\
\hline $\begin{array}{l}\text { Proportion of insectivores species with predominance of aquatic forms } \\
\text { (PSaquins) }\end{array}$ & reduces \\
\hline $\begin{array}{l}\text { Proportion of insectivores individuals with predominance of aquatic } \\
\text { forms (PNaquins) }\end{array}$ & reduces \\
\hline Proportion of detritivores species (PSdet) & increases \\
\hline Proportion of detritivores individuals (PNdet) & increases \\
\hline Proportion of onivores species (PSoni) & increases \\
\hline Proportion of onivores individuals (PNoni) & increases \\
\hline $\begin{array}{l}\text { Proportion of insectivores species with predominance of terrestrial } \\
\text { forms (PSterins) }\end{array}$ & reduces \\
\hline $\begin{array}{l}\text { Proportion of insectivores individuals with predominance of aquatic } \\
\text { forms (PNterins) }\end{array}$ & reduces \\
\hline \multicolumn{2}{|l|}{ Habitat use } \\
\hline Proportion of benthic species (PSben) & reduces \\
\hline Proportion of benthic individuals (PNben) & reduces \\
\hline Proportion of nekthonic species (PSnek) & reduces \\
\hline Proportion of nekthonic individuals (PNnek) & reduces \\
\hline
\end{tabular}

Table 4. Number of stream stretches by physical habitat index (PHI) parameter classification.

\begin{tabular}{ccccc}
\hline Habitat parameter & \multicolumn{3}{c}{ Classification } \\
\cline { 2 - 5 } & Optimal & Suboptimal & Marginal & Poor \\
\hline Epifaunal substratum/available cover & 4 & 6 & 12 & 5 \\
Velocity/depth regime & 2 & 8 & 6 & 11 \\
Channel flow status & 6 & 9 & 10 & 2 \\
Sediment deposition & 9 & 6 & 10 & 2 \\
Frequency of riffles & 4 & 2 & 15 & 6 \\
Channel alteration & 1 & 15 & 10 & 11 \\
Vegetative protection & 6 & 3 & 6 & 11 \\
Riparian vegetative zone width & 7 & 4 & 9 & 5 \\
Bank stability & 9 & 6 & 12 & 4 \\
PHI & 5 & & & \\
\hline
\end{tabular}

zone width and bank stability classified as marginal or poor. Around $75 \%$ had a frequency of riffles and vegetative protection classified as poor and marginal. The PHI ranged from 31 (poor) to 170 (optimal). About $60 \%$ of the stream stretches were classified as marginal and poor (Table 4).

We collected 2492 individuals. Five stream stretches have no species (Table 5). The total abundance $(\mathrm{N})$ metric was removed because of the low factor loading on PC1, PC2 and PC3. These axes were selected to analyse the range of metrics because they accounted for $76.82 \%$ of the total variation $(33.09,31.13$ and $12.60 \%$, respectively), higher than the broken-stick model and most metrics had the highest values on these axes. In PC3, the metrics had a load factor $>0.6$, so this axis was not analysed. Seven metrics were retained because of the responsiveness test (Table 6).

The proportion of insectivore species with a predominance of aquatic forms (PSaquins) and 
Table 5. Species richness and fishes from 27 stream stretches in the Sorocaba City (S) and FLONA-Ipanema (F) of the Medium Sorocaba River basin.

\begin{tabular}{|c|c|c|c|c|c|c|c|c|c|c|c|c|c|c|c|c|c|c|c|c|c|c|c|c|c|c|c|}
\hline species & $\bar{c}$ & สู & $\ddot{\infty}$ & ॐ & œ & ஜ & $\hat{n}$ & œ & ஜ & 응 & $\bar{s}$ & $\frac{N}{\infty}$ & $\frac{m}{\omega}$ & $\frac{5}{\infty}$ & $\frac{\infty}{\infty}$ & $\frac{0}{\infty}$ & $\frac{1}{\infty}$ & $\sum_{\infty}^{\infty}$ & $\frac{\sigma}{\infty}$ & ๙ั & స్ & สี & $\underset{\mathbb{L}}{\mathbb{4}}$ & & 10 & & ฟ \\
\hline Astyanax lacustris & $x$ & - & - & - & - & - & - & - & - & $x$ & - & - & - & $x$ & - & - & - & - & - & - & $x$ & - & $x$ & - & $x$ & $x$ & $x$ \\
\hline Australoheros facetus & - & - & - & - & - & - & - & $x$ & - & - & - & - & - & - & - & - & - & - & - & - & - & - & - & - & - & - & . \\
\hline Bryconamenricus sp & $x$ & - & - & - & - & - & - & - & $x$ & - & - & - & - & $x$ & - & - & - & - & - & - & $x$ & - & $x$ & - & - & - & $x$ \\
\hline Callichthys callichthys & - & - & - & - & - & - & - & $x$ & - & - & - & - & - & $\mathrm{X}$ & - & - & - & - & - & - & - & - & - & - & - & - & - \\
\hline Characidium zebra & - & - & - & - & - & - & - & - & - & - & - & - & - & - & - & - & - & - & - & - & - & - & - & - & - & $x$ & - \\
\hline Corydoras aeneus & - & - & - & - & - & - & - & - & - & $x$ & $x$ & - & - & - & - & - & - & - & - & - & - & - & $x$ & - & - & - & - \\
\hline Crenicichla britskii & - & - & - & - & - & - & - & - & - & - & - & - & - & - & - & - & - & - & - & - & $x$ & - & $x$ & - & - & - & - \\
\hline $\begin{array}{c}\text { Cyphocharax } \\
\text { modestus }\end{array}$ & - & - & - & - & - & - & - & - & - & $x$ & $x$ & - & - & - & - & - & - & - & - & - & - & - & $x$ & - & - & - & - \\
\hline $\begin{array}{l}\text { Deuterodon } \\
\text { intermedius }\end{array}$ & - & - & - & - & $x$ & - & - & - & - & - & - & - & - & $x$ & - & - & - & - & $x$ & $x$ & - & - & - & - & - & - & $x$ \\
\hline Geophagus brasiliensis & - & - & $x$ & - & $\mathrm{X}$ & - & - & $x$ & $x$ & $x$ & $\mathrm{x}$ & - & - & $x$ & - & - & - & - & - & - & $x$ & $x$ & - & - & - & $x$ & $x$ \\
\hline Gymnotus sylvius & - & - & - & - & - & - & - & - & $x$ & - & - & - & - & - & - & - & - & - & - & - & $x$ & - & $x$ & $x$ & - & - & $x$ \\
\hline Hoplias malabaricus & - & - & - & - & - & - & - & - & $x$ & $x$ & - & $x$ & - & - & - & - & - & - & - & - & - & - & $\mathrm{X}$ & - & - & $x$ & $x$ \\
\hline Hoplosternum littorale & - & - & - & - & - & - & - & - & - & - & - & - & - & - & - & - & - & - & - & - & - & - & $\mathrm{x}$ & - & - & - & - \\
\hline $\begin{array}{l}\text { Hyphessobrycon } \\
\text { bifasciatus }\end{array}$ & - & - & - & - & - & - & - & - & - & - & - & - & - & - & - & - & - & - & - & - & - & - & - & - & - & - & $x$ \\
\hline $\begin{array}{l}\text { Hypostomus } \\
\text { ancistroides }\end{array}$ & - & - & - & - & $x$ & - & - & $x$ & $x$ & $x$ & - & - & - & $x$ & - & - & - & - & - & $x$ & - & $x$ & - & - & - & $x$ & $x$ \\
\hline Imparfinis mirini & - & - & - & - & - & - & - & - & - & $x$ & - & - & - & $x$ & - & - & - & - & - & - & - & - & $x$ & - & - & - & - \\
\hline Oreochromis niloticus & - & - & - & - & - & - & - & $x$ & $x$ & - & - & - & - & $x$ & - & - & $x$ & - & - & - & - & $x$ & - & - & - & - & - \\
\hline Phalloceros harpagos & - & $\mathrm{x}$ & - & $x$ & $\mathrm{X}$ & - & $x$ & $x$ & $x$ & $x$ & $x$ & $\mathrm{X}$ & - & $x$ & - & - & $\mathrm{X}$ & - & $x$ & - & - & $x$ & $\mathrm{X}$ & $x$ & - & - & $x$ \\
\hline $\begin{array}{c}\text { Pimelodella } \\
\text { avanhandavae }\end{array}$ & - & - & - & - & - & - & - & - & - & $x$ & - & - & - & - & - & - & - & - & - & - & - & - & - & - & - & $x$ & - \\
\hline Poecilia reticulata & - & $x$ & - & - & $x$ & - & - & $x$ & $x$ & $x$ & $x$ & $x$ & - & $x$ & - & - & $x$ & - & $x$ & - & - & $x$ & - & $x$ & - & - & - \\
\hline Prochilodus lineatus & - & - & - & - & - & - & - & - & - & - & - & - & - & - & - & - & - & - & - & - & - & - & $x$ & - & - & - & - \\
\hline Psalidodon bockmanni & $x$ & - & - & - & - & - & - & - & - & - & - & - & - & $x$ & - & - & - & - & - & - & - & - & $x$ & - & - & $x$ & - \\
\hline Psalidodon fasciatus & - & - & $x$ & - & $x$ & - & - & - & $x$ & $x$ & - & - & - & - & - & - & - & - & - & - & - & $x$ & $x$ & $x$ & $x$ & - & - \\
\hline Rhamdia quelen & - & - & - & - & $\mathrm{X}$ & - & - & - & - & $x$ & - & - & - & - & - & - & - & - & - & - & - & - & $\mathrm{X}$ & - & - & $x$ & - \\
\hline Serrapinnus notomelas & - & - & - & - & - & - & - & - & - & - & - & - & - & - & - & - & - & - & - & - & - & - & $x$ & $x$ & - & - & - \\
\hline $\begin{array}{c}\text { Synbranchus } \\
\text { marmoratus }\end{array}$ & - & - & - & - & - & - & - & - & $x$ & $x$ & - & $x$ & - & - & - & - & $x$ & - & - & - & $x$ & - & - & - & - & - & - \\
\hline Species richness & 3 & 2 & 2 & 1 & 7 & 0 & 1 & 7 & 10 & 13 & 5 & 4 & 0 & 11 & 0 & 0 & 4 & 0 & 3 & 2 & 6 & 6 & 15 & 5 & 2 & 8 & 9 \\
\hline
\end{tabular}

proportion of benthic species (PSben) was considered redundant with species richness $(\mathrm{S})$. The proportion of insectivore individuals with a predominance of aquatic forms (PNaquins) was considered redundant with the proportion of benthic individuals (PNben).

Four metrics were finally included in the MFI. They belonged to the categories richness and origin (S), abundance (PCyp), trophic structure (Stroph) and habitat use (PNben) (Table 7). Five streams $(18 \%)$ were classified as reference or good and $16(60 \%)$ as poor or bad (Table 8$)$.

Five candidate metrics were significantly correlated with the MFI: Simpson effective number of species $(1 / \mathrm{D}, r=0.76)$, Shannon effective number of species $\left(\mathrm{e}^{\wedge} \mathrm{H}, r=0.82\right)$, proportion of omnivore species and individuals (PSoni, $r=-0.48$ and PNoni, $\mathrm{r}=-0.47$ ) and proportion of insectivores individuals with predominance of aquatic forms (PNaquins, $r=0.70$ ).

\section{Discussion}

Urbanization altered many aspects of fish biological integrity. We found a low EQR (MFI) at $60 \%$ of the stretches. Three reference stretches can be used for a physical restoration proposal, such as epifaunal substratum/available cover, velocity/depth regime, riparian vegetative zone width, bank stability, frequency of riffles and vegetative protection. The MFI used four biological variables, and the low number of metrics contributes to a quick and easy biomonitoring process (Gonino et al., 2020). These variables allowed for a differentiation between sites with different impact levels in the urban area. The selected metrics are easy estimates to obtain and proved to be satisfactory for the adaptation of the MFI.

Species richness proved to be an important metric and can be used to infer urban streams biotic integrity. As it is a very intuitive variable (Magurran, 2004), species richness has been widely used to infer ecological systems quality (Roth et al., 2000). The distribution of the number of individuals per species, represented by dominance, was not correlated with environmental degradation despite being a helpful indicator of changes in the stream fish biotic integrity (Terra et al., 2013). On the 
Table 6. Minimum (Min), maximum (Max), mean and coefficient of variation (CV) of the candidate metrics (see Table 3) for the multimetric fish index (MFI). Range and responsiveness significance: PCA metric loadings (PC1 and PC2) and Pearson correlation coefficient with PHI (PHIr).

\begin{tabular}{cccccccc}
\hline & Min & Max & Mean & CV & PC 1 & PC 2 & PHIr \\
\hline S & 0 & 15 & 5.73 & 0.69 & 0.83 & - & 0.48 \\
$1 / D$ & 1 & 6.87 & 2.55 & 0.63 & 0.77 & - & - \\
$\mathrm{e}^{\wedge} \mathrm{H}$ & 1 & 8.38 & 3.23 & 0.65 & 0.82 & - & - \\
Psexot & 0 & $50 \%$ & $15 \%$ & 1.14 & - & 0.79 & - \\
$N$ & 4 & 977 & 113.27 & 1.92 & - & - & - \\
Pnexot & 0 & $33 \%$ & $10 \%$ & 1.22 & - & 0.76 & - \\
PCyp & 0 & $100 \%$ & $46 \%$ & 0.92 & -0.66 & 0.70 & -0.46 \\
Stroph & 1 & 6 & 3.27 & 0.42 & 0.84 & - & 0.36 \\
PSaquins & 0 & $38 \%$ & $11 \%$ & 1.23 & 0.78 & - & 0.51 \\
PNaquins & 0 & $70 \%$ & $9 \%$ & 2.15 & 0.61 & - & 0.44 \\
PSdet & 0 & $50 \%$ & $20 \%$ & 0.81 & - & 0.67 & - \\
PNdet & 0 & $31 \%$ & $13 \%$ & 0.88 & - & 0.67 & - \\
PSoni & 0 & $100 \%$ & $44 \%$ & 0.54 & -0.67 & - & - \\
PNoni & 0 & $100 \%$ & $51 \%$ & 0.58 & -0.65 & - & - \\
PSterins & 0 & $50 \%$ & $18 \%$ & 1.00 & - & -0.66 & - \\
Pnterins & 0 & $94 \%$ & $26 \%$ & 1.24 & - & -0.87 & - \\
PSben & 0 & $60 \%$ & $26 \%$ & 0.88 & 0.66 & - & 0.55 \\
PNben & 0 & $73 \%$ & $17 \%$ & 1.26 & 0.71 & - & 0.58 \\
PSnek & 0 & $100 \%$ & $27 \%$ & 1.06 & - & -0.82 & - \\
PNnek & 0 & $100 \%$ & $33 \%$ & 1.13 & - & -0.88 & - \\
\hline
\end{tabular}

Table 7. Multimetric fish index (MFI) metrics (see Table 3) and class boundaries for Sorocaba City streams.

\begin{tabular}{cccccc}
\hline Metric & Reference & Good & Moderate & Poor & Bad \\
\hline S & $>11$ & 9 to 10 & 6 to 8 & 3 to 5 & $<2$ \\
PCyp & $<10 \%$ & 10 to $30 \%$ & 30 to $45 \%$ & $45 \%$ to $70 \%$ & $>70 \%$ \\
STroph & $>5$ & 4 & 3 & 2 & 1 \\
Pben & $>70 \%$ & 40 to $70 \%$ & 30 to $40 \%$ & 20 to $30 \%$ & $<20 \%$ \\
\hline
\end{tabular}

Table 8. Detailed descriptions of stream multimetric fish index (MFI) with ecological quality ratio (EQR) values and number of streams by category $(n)$.

\begin{tabular}{|c|c|c|c|}
\hline Categories & MFI & Description & $n$ \\
\hline Reference & $\geq 0.8$ & $\begin{array}{l}\text { Above eight species, low proportion of Cyprinodontiformes, more than four } \\
\text { trophic groups, and high abundance of benthic species }\end{array}$ & 3 \\
\hline Good & $\geq 0.6<0.8$ & $\begin{array}{l}\text { Above eight species, low proportion of Cyprinodontiformes, more than four } \\
\text { trophic groups, and moderate abundance of benthic species }\end{array}$ & 2 \\
\hline Moderate & $\geq 0.4<0.6$ & $\begin{array}{l}\text { Species richness of six to eight species, moderate abundance of } \\
\text { Cyprinodontiformes, up to three trophic groups, and presence of benthic species }\end{array}$ & 6 \\
\hline Poor & $\geq 0.2<0.4$ & $\begin{array}{l}\text { Species richness around four species, moderate to high abundance of } \\
\text { Cyprinodontiformes, up to two trophic groups, and low presence of benthic species }\end{array}$ & 5 \\
\hline Bad & $<0.2$ & $\begin{array}{c}\text { Species richness up to two species, high abundance of Cyprinodontiformes, one } \\
\text { trophic group, low presence of benthic species or repeated samplings without } \\
\text { catching any fishes }\end{array}$ & 11 \\
\hline
\end{tabular}

other hand, Bozzetti \& Schulz (2004) and Esteves $\&$ Alexandre (2011) verified that low IBI scores and diversity indices reflected environmental degradation. The proportion of exotic species was not a good metric. It was not correlated with MFI, possibly due to the capture of only two species that may have caused a low gradient of values capable of indicating biotic integrity.

The proportion of Cyprinodontiformes abundance (PCyp) is relevant in the lower biotic integrity stream classification. In this order, we captured two species, a native Phalloceros harpagos Lucinda, 2008 and an exotic Poecilia reticulata Peters, 1859. Poecilia reticulata is characterized by its small size, wide geographical distribution, and large capacity to live in low environmental quality environments (Casatti et al., 2009; Cunico et al., 2006). Esteves and Alexandre (2011) selected the abundance of Astyanax lacustris as a metric to compose the IBI. This species was more 
abundant in impaired urban sites than less degraded sites.

Trophic specialization is not a feature of tropical fishes (Abelha et al., 2001). We identified six types of eating habits. These six types of eating habit have allowed us to obtain a range that can detect reference and good or bad biotic integrity. Streams with many eating habits reflect the amplitude of food chain levels and trophic relationships (Uieda et al., 1997; Casatti et al., 2006). In developing an IBI based on fish communities to assess the effects of rural and urban land use on a Paraná River basin stream, Esteves \& Alexandre (2011) selected richness of insectivore and detritivore-algivore species that increased with environmental quality.

The contribution of insectivores may reflect food availability and not food specialization (Abelha et al., 2001). The increase in proportional insectivores contribution that feeds predominantly on aquatic insects is related to greater biotic and environmental integrity environments (Cetra \& Ferreira, 2016). Marciano et al. (2004) indicated some degree of degradation using the abundance of invertivores in third-order streams in the Sorocaba river basin using an IBI composed of richness, total abundance of the species, intolerance, and trophic guilds. On the other hand, omnivores representing more generalist species in the food chain were not part of the MFI. This group is not statistically correlated with physical stream integrity but is negatively correlated with biotic integrity. Therefore, high values of the proportion of omnivorous individuals and species represent low biotic integrity.

Ferreira \& Casatti (2006) verified that habitat structure measured by a PHI index influenced the stream biotic integrity assessed by fish assemblages in the Upper Rio Paraná basin. The low physical integrity of urban streams caused by fine sediments entry gives rise to the loss of substratum-associated habitats complexity. This situation can reduce surface water and groundwater exchange and potentially decrease the hyporheic zone size and function (O'Driscoll et al., 2010). In this sense, the increase in the proportion of benthic individuals is a metric that indicates good biotic integrity. Casatti et al. (2012) recorded in streams with riparian zone preserved an ichthyofauna with specialized habits, notably benthic insectivores, intolerant, and rheophilics. Santos \& Esteves (2015) verified that the IBI successfully detected the effects of different riparian conditions on stream fish fauna, suggesting the riparian zone was essential to maintaining ecosystem integrity in the intensively managed sugarcane areas.

Initial stream restoration records were collected in the 19th century in the U.S.A., Germany, and Norway with fishing records (Roni \& Beechie, 2013). There are no physical stream restoration projects in urban areas in Brazil. Strategies for restoration in urban streams for many years have not solved problems related to excess sediment, loss of riparian vegetation and habitat diversity structures (Wenger et al., 2009). The catchment level restoration approach seems to be the most efficient as it has an ecosystem approach (Roni \& Beechie, 2013). Therefore, we propose adopting this MFI as an indicator of the effect of a more holistic restoration, and we believe in the success of this approach as we have found streams around the city with good environmental and biotic quality (Gonino et al., 2020).

\section{Acknowledgements}

We are grateful to Dr. George M.T. Mattox (UFSCar/Sorocaba) for species identification. The Fundação de Amparo à Pesquisa do Estado de São Paulo (FAPESP) for financial support (Proc. 2017/25860-3). We are grateful to Rodrigo Almeida da Silva, José Miguel Arcanjo da Silva, Douglas Sérgio Bueno da Silva and Rayssa Bernardi Guinato from the UFSCar during data collection.

\section{References}

ABELHA, M.C.F., AGOSTinho, A.A. and GOULART, E. Plasticidade trófica em peixes de água doce. Acta Scientiarum, 2001, 23, 425-434.

ABILHOA, V. Aspectos da história natural de Astyanax scabripinnis Jenyns (Teleostei, Characidae) em um riacho de floresta com araucária no sul do Brasil. Revista Brasileira de Zoologia, 2007, 24(4), 997-1005. http://dx.doi.org/10.1590/S010181752007000400016.

BARBOUR, M.T., GERRITSEN, J., SNYDER, B.D. and STRIBLING, J.B. Rapid bioassessment protocols for use in streams and wadeable rivers: periphyton, benthic macroinvertebrates and fish. 2 nd ed. Washington: US Environmental Protection Agency, Office of Water; 1999. EPA 841-B-99-002.

BÖHMER, J., RAWER-JOST, C., ZENKER, A., MEIER, C., FELD, C.K., BISS, R. and HERING, D. Assessing streams in Germany with benthic invertebrates: development of a multimetric invertebrate based assessment system. Limnologica, 2004, 34(4), 416-432. http://dx.doi.org/10.1016/ S0075-9511(04)80010-0. 
BOZZETTI, M. and SCHULZ, U.H. An index of biotic integrity based on fish assemblages for subtropical streams in southern Brazil. Hydrobiologia, 2004, 529(1), 133-144. http://dx.doi.org/10.1007/s10750004-5738-6.

BRANDÃO-GONÇALVES, L., OLIVEIRA, S.A. and LIMA-JUNIOR, S.E. Hábitos alimentares da ictiofauna do córrego Franco, Mato Grosso do Sul, Brasil. Biota Neotropica, 2010, 10(2), 21-30. http:// dx.doi.org/10.1590/S1676-06032010000200001.

CASATTI, L., DE PAULA FERREIRA, C. and CARVALHO, F.R. Grass-dominated stream sites exhibit low fish species diversity and dominance by guppies: an assessment of two tropical pasture river basins. Hydrobiologia, 2009, 632(1), 273-283. http:// dx.doi.org/10.1007/s10750-009-9849-y.

CASATTI, L., LANGEANI, F. and FERREIRA, C.P. Effects of physical habitat degradation on the stream fish assemblage structure in a pasture region. Environmental Management, 2006, 38(6), 974-982. http://dx.doi.org/10.1007/s00267-005-0212-4. PMid:16990983.

CASATTI, L., TERESA, F.B., GONÇALVES-SOUZA, T., BESSA, E., MANZOTTI, A.R., GONÇALVES, C.S. and ZENI, J.O. From forests to cattail: how does the riparian zone influence stream fish? Neotropical Ichthyology, 2012, 10(1), 205-214. http://dx.doi. org/10.1590/S1679-62252012000100020.

CETRA, M. and FERREIRA, F.C. Índice de Integridade Biótica utilizando a comunidade de peixes para riachos de cabeceira que cruzam a Mata Atlântica do sul do estado de São Paulo, Brasil. Acta Limnologica Brasiliensia, 2016, 28, e22.

CUNICO, A.M., AGOSTINHO, A.A. and LATINI, J.D. Influência da urbanização sobre as assembléias de peixes em três córregos de Maringá, Paraná. Revista Brasileira de Zoologia, 2006, 23(4), 1101-1110. http:// dx.doi.org/10.1590/S0101-81752006000400018.

CZEGLÉDI, I., KERN, B., TÓTH, R., SERESS, G. and ERÖS, T. Impacts of urbanization on stream fish assemblages: the role of the species pool and the local environment. Frontiers in Ecology and Evolution, 2020, 8, 1-10. http://dx.doi.org/10.3389/ fevo.2020.00137.

DAVIS, W.S. and SIMON, T.P. Biological assessment and criteria: tools for water resource planning and decision making. Boca Raton: CRC Press, 1995.

ESTEVES, K.E. and ALEXANDRE, C.V. Development of an index of biotic integrity based on fish communities to assess the effects of rural and urban land use on a stream in southeastern Brazil. International Review of Hydrobiology, 2011, 96(3), 296-317. http:// dx.doi.org/10.1002/iroh.201111297.

FAME CONSORTIUM. Manual for the application of the European Fish Index - EFI. A fish-based method to assess the ecological status of European rivers in support of the Water Framework Directive, 2004, Version 1.1. UK: Fame Consortium, 2004.

FERNÁNDEZ, E., FERRIZ, R., BENTOS, C. and LÓPEZ, G.R. Dieta y ecomorfología de la ictiofauna del Arroyo Manantiales, provincia de Buenos Aires, Argentina. Revista del Museo Argentino de Ciencias Naturales, 2012, 14, 1-13. http://dx.doi. org/10.22179/REVMACN.14.206.

FERREIRA, C.D.P. and CASATTI, L. Integridade biótica de um córrego na bacia do Alto Rio Paraná avaliada por meio da comunidade de peixes. Biota Neotropica, 2006, 6(3), 1-25.

GONINO, G., BENEDITO, E., CIONEK, V.D.M., FERREIRA, M.T. and OLIVEIRA, J.M. A FishBased Index of Biotic Integrity for Neotropical Rainforest Sandy Soil Streams-Southern Brazil. Water (Basel), 2020, 12(4), 1215. http://dx.doi. org/10.3390/w12041215.

HELMS, B.S., FEMINELLA, J.W. and PAN, S. Detection of biotic responses to urbanization using fish assemblages from small streams of western Georgia, USA. Urban Ecosystems, 2005, 8(1), 3957. http://dx.doi.org/10.1007/s11252-005-1418-1.

HERING, D., FELD, C.K., MOOG, O. and OFENBÖCK, T. Cook book for the development of a Multimetric Index for biological condition of aquatic ecosystems: Experiences from the European AQEM and STAR projects and related initiatives. Hydrobiologia, 2006, 566(1), 311-324. http://dx.doi. org/10.1007/s10750-006-0087-2.

HUGHES, R.M., KAUFMANN, P.R., HERLIHY, A.T., KINCAID, T.M., REYNOLDS, L. and LARSEN, D.P. A process for developing and evaluating indices of fish assemblage integrity. Canadian Journal of Fisheries and Aquatic Sciences, 1998, 55(7), 16181631. http://dx.doi.org/10.1139/f98-060.

INSTITUTO BRASILEIRO DE GEOGRAFIA E ESTATÍSTICA - IBGE. Censo 2010 [online]. Rio de Janeiro: IBGE; 2010 [viewed 20 June 2019]. Available from: https://censo2010.ibge.gov.br

JIA, Y., SUI, X.Y. and CHEN, Y.F. Development of a fishbased index of biotic integrity for wadeable streams in Southern China. Environmental Management, 2013, 52(4), 995-1008. http://dx.doi.org/10.1007/ s00267-013-0129-2. PMid:23892683.

KARR, J.R. Assessment of biotic integrity using fish communities. Fisheries (Bethesda, Md.), 1981, 6(6), 21-27. http://dx.doi.org/10.1577/15488446(1981)006<0021:AOBIUF>2.0.CO;2.

KARR, J.R. Biological integrity: a long-neglected aspect of water resource management. Ecological Applications, 1991, 1(1), 66-84. http://dx.doi. org/10.2307/1941848. PMid:27755684.

LANGEANI, F., CASTRO, R.M.C., OYAKAWA, O.T., SHIBATTA, O.A., PAVANELLI, C.S. and CASATTI, L. Diversidade da ictiofauna do Alto 
Rio Paraná: composição atual e perspectivas futuras. Biota Neotropica, 2007, 7(3), 181-197. http://dx.doi. org/10.1590/S1676-06032007000300020.

MAGURRAN, A.E. Measuring biological diversity. 2nd ed. Oxford: Blackwell Publishing, 2004, pp. 256.

MARCIANO, F.T., CHAUDHRY, F.H. and RIBEIRO, M.C.L.B. Evaluation of the index of biotic integrity in the Sorocaba River Basin (Brazil, SP) based on fish communities. Acta Limnologica Brasiliensia, 2004, 16, 225-237.

MELLO, K., TOPPA, R.H. and CARDOSO-LEITE, E. Priority areas for forest conservation in an urban landscape at the transition between Atlantic Forest and Cerrado. Cerne, 2016, 22(3), 277-288. http:// dx.doi.org/10.1590/01047760201622032172.

MORAES, M.F.P.G., BARBOLA, I.F. and GUEDES, É.A.C. Barbola. I.F. and Guedes, E.A.C. Alimentação e relaçóes morfológicas com o aparelho digestivo do "curimbatá", Prochilodus lineatus (Valenciennes) (Osteichthyes, Prochilodontidae), de uma lagoa do sul do Brasil. Revista Brasileira de Zoologia, 1997, 14(1), 169-180. http://dx.doi.org/10.1590/S010181751997000100015 .

MORGAN, R.P. and CUSHMAN, S.F. Urbanization effects on stream fish assemblages in Maryland, USA. Journal of the North American Benthological Society, 2005, 24(3), 643-655. http://dx.doi. org/10.1899/04-019.1.

O’DRISCOLL, M., CLINTON, S., JEFFERSON, A., MANDA, A. and MCMILLAN, S. Urbanization effects on watershed hydrology and in-stream processes in the Southern United States. Water (Basel), 2010, 2(3), 605-648. http://dx.doi.org/10.3390/ w2030605.

OMERNIK, J.M. Ecoregions: a framework for managing ecosystems. The George Wright Forum, 1995, 12, 35-50.

PAUL, M.J. and MEYER, J.L. Streams in the urban landscape. Annual Review of Ecology and Systematics, 2001, 32(1), 333-365. http://dx.doi.org/10.1146/ annurev.ecolsys.32.081501.114040.

PEASE, A.A., GONZALEZ-DIAZ, A.A., RODILESHERNANDEZ, R. and WINEMILLER, K.O. Functional diversity and trait-environment relationships of stream fish assemblages in a large tropical catchment. Freshwater Biology, 2012, 57(5), 1060-1075. http://dx.doi.org/10.1111/j.13652427.2012.02768.x.

RODRÍGUEZ, M.A. and LEWIS JUNIOR, W.M. Structure of fish assemblages along environmental gradients in floodplain lakes of the Orinoco River. Ecological Monographs, 1997, 67(1), 109-128. http:// dx.doi.org/10.1890/0012-9615(1997)067[0109:SO FAAE]2.0.CO;2.

RONI, P. and BEECHIE, T. Stream and watershed restoration: a guide to restoring riverine processes and habitats. England: Wiley-Blackwell, 2013, pp. 300.
ROTH, N.E., SOUTHERLAND, M.T., CHAILLOU, J.C., KAZYAK, P.F. and STRANKO, S.A. Refinement and validation of a fish index of biotic integrity for Maryland Streams. Annapolis: Maryland Department of Natural Resources, 2000.

SANTOS, F.B. and ESTEVES, K.E. A fish-based index of biotic integrity for the assessment of streams located in a sugarcane-dominated landscape in southeastern Brazil. Environmental Management, 2015, 56(2), 532-548. http://dx.doi.org/10.1007/ s00267-015-0516-y. PMid:25924789.

SHELDON, F., LEIGH, C., NEILAN, W., NEWHAM, M., POLSON, C. and HADWEN, W. Urbanization: hydrology, water quality, and influences on ecosystem health. In: A.K. SHARMA, T. GARDNER and D. BEGBIE, eds. Approaches to water sensitive urban design. USA: Elsevier Sicence, 2019, pp. 229-248.

SMITH, W.S. Os peixes do rio Sorocaba: a história de uma bacia hidrográfica. Sorocaba: Editora TCM Comunicação, 2003.

TERRA, B.F., HUGHES, R.M., FRANCELINO, M.R. and ARAÚJO, F.G. Assessment of biotic condition of Atlantic Rain Forest streams: a fish-based multimetric approach. Ecological Indicators, 2013, 34, 136-148. http://dx.doi.org/10.1016/j.ecolind.2013.05.001.

UIEDA, V.S., BUZZATO, P. and KIKUCHI, R.M. Partilha de recursos alimentares em peixes em um riacho de serra do Sudeste do Brasil. Anais da Academia Brasileira de Ciências, 1997, 69, 243-252.

UNITED STATES ENVIRONMENTAL PROTECTION AGENCY - USEPA. Causal Analysis/Diagnosis Decision Information System (CADDIS). USA: USEPA. 2017 [viewed 5 June 2019]. Available from: www.epa.gov/caddis

WALSH, C.J., FLETCHER, T.D. and LADSON, A.R. Stream restoration in urban catchments through redesigning stormwater systems: looking to the catchment to save the stream. Journal of the North American Benthological Society, 2005, 24(3), 690-705. http://dx.doi.org/10.1899/04-020.1.

WANG, L., LYONS, J., KANEHL, P. andBANNERMAN, R. Impacts of urbanization on stream habitat and fish across multiple spatial scales. Environmental Management, 2001, 28(2), 255-266. http://dx.doi. org/10.1007/s0026702409. PMid:11443388.

WENGER, S.J., ROY, A.H., JACKSON, C.R., BERNHARDT, E.S., CARTER, T.L., FILOSO, S., GIBSON, C.A., HESSION, W.C., KAUSHAL, S.S., MARTI, E., MEYER, J.L., PALMER, M.A., PAUL, M.J., PURCELL, A.H., RAMIREZ, A., ROSEMOND, A.D., SCHOFIELD, K.A., SUDDUTH, E.B. and WALSH, C.J. Twenty-six key research questions in urban stream ecology: an assessment of the state of the science. Journal of the North American Benthological Society, 2009, 28(4), 1080-1098. http://dx.doi.org/10.1899/08-186.1. 
WILLIS, S.C., WINEMILLER, K.O. and LOPEZFERNANDEZ, H. Habitat structural complexity and morphological diversity of fish assemblages in a Neotropical floodplain river. Oecologia, 2005, 142(2), 284-295. http://dx.doi.org/10.1007/s00442-0041723-z. PMid:15655689.

WINEMILLER, K.O., AGOSTINHO, A.A. and CARAMASCHI, E.P. Fish ecology in tropical streams.
In: D. DUDGEON, ed. Tropical stream ecology. Cambridge: Academic Press, 2018, pp. 107-146.

Received: 19 May 2020 Accepted: 20 May 2021

Associate Editors: Irineu Bianchini Junior, Antonio Fernando Monteiro Camargo. 